\title{
Cooperative interactions govern the fermiology of the polar metal $\mathrm{Ca}_{3} \mathrm{Ru}_{2} \mathrm{O}_{7}$
}

\author{
Danilo Puggioni $\odot,{ }^{1, *}$ M. Horio $\odot,{ }^{2}$ J. Chang $\odot,{ }^{2}$ and James M. Rondinelli $\odot{ }^{1, \dagger}$ \\ ${ }^{1}$ Department of Materials Science and Engineering, Northwestern University, Evanston, Illinois 60208, USA \\ ${ }^{2}$ Physik-Institut, Universität Zürich, Winterthurerstrasse 190, CH-8057 Zürich, Switzerland
}

(Received 4 March 2020; accepted 16 April 2020; published 8 May 2020)

\begin{abstract}
The antiferromagnetic Ruddlesden-Popper ruthenate $\mathrm{Ca}_{3} \mathrm{Ru}_{2} \mathrm{O}_{7}$ is a model polar metal, combining inversion symmetry breaking with metallic conductivity; however, its low-temperature $(T<48 \mathrm{~K})$ crystal structure and Fermi-surface topology remain ambiguous despite numerous measurements and theoretical studies. Here we perform both first-principles calculations with static correlations and angle-resolved photoelectron spectroscopy experiments to construct a complete model of $\mathrm{Ca}_{3} \mathrm{Ru}_{2} \mathrm{O}_{7}$, reconciling inconsistencies among interpretations of electrical transport, thermopower measurements, and momentum- and energy-resolved band dispersions. The solution relies on treating the interplay among Coulomb repulsion, magnetic ordering, spin-orbit interactions, and the $\mathrm{RuO}_{6}$ octahedral degrees of freedom on equal footing. For temperatures $30<T<48 \mathrm{~K}$, we propose weak electron-electron interactions produce a symmetry-preserving metal-semimetal transition with Weyl nodes in proximity to the Fermi level, whereas an orthorhombic $P n 2_{1} a$ structure emerges for $T<30 \mathrm{~K}$, exhibiting charge- and spin-density waves from enhanced Coulombic interactions.
\end{abstract}

DOI: 10.1103/PhysRevResearch.2.023141

\section{INTRODUCTION}

Ruddlesden-Popper $\mathrm{Ca}_{3} \mathrm{Ru}_{2} \mathrm{O}_{7}$ is a unique polar metal exhibiting a rich phase diagram (Fig. 1), colossal magnetoresistance [2,3], highly anisotropic electrical resistivity [4], and polar domain structures [5]. It displays multiple magnetic and electronic transitions without changes in crystal symmetry, making it also a member of a rare condensed-matter family exhibiting isosymmetric transitions [6]. $\mathrm{Ca}_{3} \mathrm{Ru}_{2} \mathrm{O}_{7}$ exhibits the polar space group $B b 2_{1} m$ from room temperature to $8 \mathrm{~K}$ [1] owing to in-phase and out-of-phase $\mathrm{RuO}_{6}$ octahedral rotations along the [001] and [110] directions, respectively [5]. Below $T_{N}=56 \mathrm{~K}$, it exhibits antiferromagnetic order (AFMa) characterized by ferromagnetic perovskite bilayers with a [100] easy axis, i.e., the short axis of the crystal. The bilayers then couple antiferromagnetically along [001]. At $T_{s}=48 \mathrm{~K}$, it undergoes a first-order isosymmetric transition marked by a discontinuous change in lattice parameters but no change in occupied Wyckoff positions or space group. Interestingly, below $T_{s}$, the lattice constants are nearly temperature independent [1], despite an electrical resistivity transition from a three- to two-dimensional state. Coincident with $T_{s}$, a second magnetic transition occurs, AFM- $a \rightarrow \mathrm{AFM}-b$; the easy axis switches from the [100] to [010] direction [Fig. 2(a)]. Understanding how multiple orders harmonize and evolve with broken inversion and time-reversal symmetries as in

\footnotetext{
*danilo.puggioni@northwestern.edu

†jrondinelli@northwestern.edu

Published by the American Physical Society under the terms of the Creative Commons Attribution 4.0 International license. Further distribution of this work must maintain attribution to the author(s) and the published article's title, journal citation, and DOI.
}

$\mathrm{Ca}_{3} \mathrm{Ru}_{2} \mathrm{O}_{7}$ is critical for control of topological quasiparticle excitations [7].

Angle-resolved photoelectron spectroscopy (ARPES) and optical-pump and optical-probe experiments propose an electronic instability underlies the transport anisotropy at $T_{s}$ by removal of well-defined electron- and holelike pockets on the Fermi surface (FS) upon cooling [8-11]. The electronlike band about the $\Gamma$ point is removed, and asymmetric FS changes occur at the zone boundary [11]: A temperaturedependent electron pocket at $k=M(\pi / a, 0)$ shrinks upon cooling through $T=30 \mathrm{~K}$, while a temperature-independent hole pocket is found at $M^{\prime}(0, \pi / b)$. These data suggest either a FS reconstruction [11] or the appearance of a charge density wave at $T_{s}$ [12], respectively; however, there remains no direct evidence for its presence in $\mathrm{Ca}_{3} \mathrm{Ru}_{2} \mathrm{O}_{7}$. Below $30 \mathrm{~K}$, quasi-two-dimensional (quasi-2D) electron and hole pockets (areas $\approx 0.3 \%$ of the Brillouin zone) survive and form the lowtemperature FS as determined from quantum oscillations [8].

In contrast, thermoelectric measurements show strong asymmetric FS changes with temperature based on assignments of the principal carrier type with the sign of the thermopower coefficient along the $a$ and $b$ crystal axes [13]. This transport-deduced evolution is inconsistent with the aforementioned optics. Above $T_{s}$, only holelike bands are reported, whereas only electroniclike bands contribute for intermediate temperatures, $30<T<48 \mathrm{~K}$, in disagreement with the ARPES-resolved electron-pocket dependencies about $k=M(\pi / a, 0)$. Below $T=30 \mathrm{~K}$, however, negative and positive thermopower responses were measured along the crystallographic $a$ and $b$ axes, respectively, indicating predominately electron- and holelike bands along these directions on the FS-features more consistent with ARPES.

Interestingly, all previous band-structure calculations based on density-functional theory (DFT) $[14,15]$ are also 


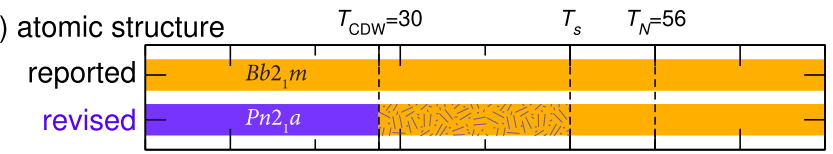

(b) physical properties

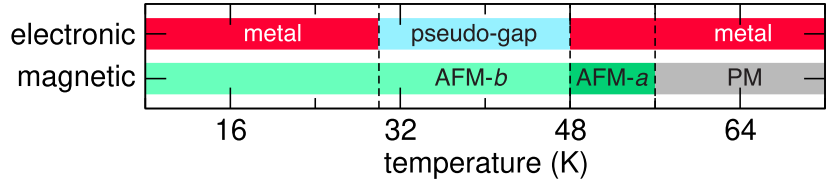

FIG. 1. Low-temperature phase diagram of $\mathrm{Ca}_{3} \mathrm{Ru}_{2} \mathrm{O}_{7}$, which assigns isosymmetric (symmetry-preserving) transitions to the magnetic $\left(T_{N}\right.$ and $T_{s}$ ) and electronic $T_{\mathrm{CDW}}$ transitions according to Ref. [1]. The revised diagram replaces the $B b 2_{1} m$ symmetry with $P n 2_{1} a$ for $T<T_{\mathrm{CDW}}$; the shaded region indicates that $P n 2_{1} a$ may persist to $T_{s}$.

inconsistent with the ARPES [8,9,11] and thermoelectric transport measurements [13]. This is unusual given that DFT methods typically provide accurate descriptions of weakly correlated metals [16-18]. This discrepancy may be due, in part, to underlying structural model assumptions deduced on the reported nearly temperature independent changes in the atomic structure below $T_{s}$ and difficulties in resolving weak reflections in layered perovskites [19,20].

Which interactions cause the change in the electronic structure at $T_{s}=48 \mathrm{~K}$ and $T=30 \mathrm{~K}$ and how one can reconcile the aforementioned discrepancies remain unclarified. Here we provide a model for the low-temperature phases of $\mathrm{Ca}_{3} \mathrm{Ru}_{2} \mathrm{O}_{7}$ based on a single-particle DFT plus Hubbard $U$ description, which is fully consistent with all available data. For all values of the Coulomb interaction $U$, we find a Van Hove singularity (VHS) within $\lesssim 20 \mathrm{meV}$ of the Fermi level owing to static Coulomb interactions that enhance broken band degeneracies near $k=X(\pi / a, \pi / b)$ split by the spin-orbit interaction (SOI). The filling of these states occurs proximate to Weyl points in momentum space that arise from broken inversion and time-reversal symmetries. Thus, the FS comprises both electron- and holelike pockets; however, the VHS prohibits using thermopower data alone to deduce the evolution of these bands. We use this electronic structure to propose that a Lifshitz-like electronic transition first occurs at $T_{s}=48 \mathrm{~K}$ for $U<1.3 \mathrm{eV}$ and a gapped-out FS persists for $30<T<$ $48 \mathrm{~K}$ within the known $B b 2{ }_{1} m$ structure. Next, upon increasing the value of $U$ (cooling below $30 \mathrm{~K}$ ), we stabilize a unique low-temperature phase $\left(P n 2_{1} a\right)$ with charge-and spindensity waves that support conclusions of earlier optical spectroscopy experiments [12]. Finally, we compare our $T \leqslant 30 \mathrm{~K}$ band structure calculations for $P n 2_{1} a \mathrm{Ca}_{3} \mathrm{Ru}_{2} \mathrm{O}_{7}$ with direct ARPES data to confirm both the existence of linear band dispersions below the Fermi level $(15 \mathrm{meV})$ at $M(\pi / a, 0)$ and the fact that enhanced interactions $(U=1.6 \mathrm{eV})$ with both SOI and $\mathrm{RuO}_{6}$ distortions are required to describe the fermiology. Thus, we propose the ground-state crystal structure is $P n 2_{1} a$ and that this phase may persist above $T=30 \mathrm{~K}$ and below $T_{s}=48 \mathrm{~K}$.

\section{MODEL AND METHODS}

We formulate a model that explicitly accounts for the interplay among Coulomb repulsion, magnetic ordering, SOI, and the structural degrees of freedom - a combination of features that has not been previously pursued. All previous DFT studies used the reported experimental structure [1], neglecting atomic relaxations $[9,14,15]$. We include static correlations through a local Coulomb interaction $U$, which is screened through a temperature-dependent electronic bandwidth $W$ as $U / W(T)$ and further stabilizes states with orbital degeneracies broken by the SOI. We justify this approach as follows: First, at $T_{s}$, the $c$-axis compression increases the $\mathrm{RuO}_{6}$ octahedral rotational angles, which are known to narrow the bandwidth (reduce $W$ ) of the low-energy $t_{2 g}$ orbitals in oxides comprising octahedral $\mathrm{Ru}^{4+}$. These angles exhibit a further deviation from $180^{\circ}$ upon cooling [1]. Second, finite temperature will effectively reduce the local interaction allowing double occupancy of the $d$ manifold, increasing toward a noninteracting value at high temperature [21]. Thus, the effective $U$ at room temperature will be smaller than that at $0 \mathrm{~K}$.

We perform first-principles DFT $+U$ calculations [22] and vary the on-site Coulomb repulsion from 0 to $2 \mathrm{eV}$ within the $\mathrm{Ru} 4 d$ manifold. This upper limit on $U$ is close to the constrained random-phase approximation and dynamical mean-field theory values for the related ruthenate $\mathrm{Ca}_{2} \mathrm{RuO}_{4}$ [23-25]. We then interpret these dependencies and assign them to transitions at $T_{s}=48 \mathrm{~K}$ and $T=30 \mathrm{~K}$. Previous
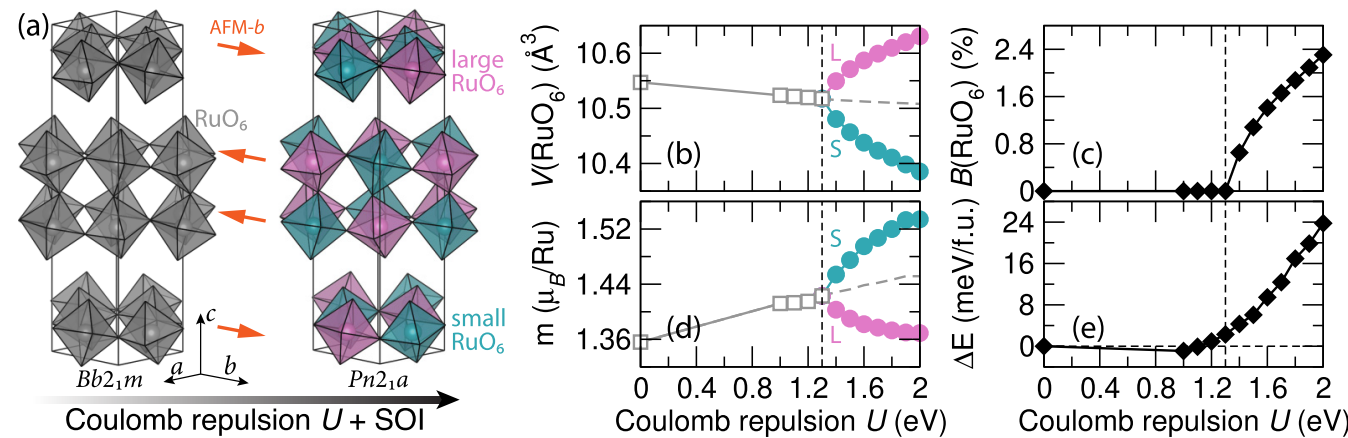

FIG. 2. (a) Crystal structure of $\mathrm{Ca}_{3} \mathrm{Ru}_{2} \mathrm{O}_{7}$ as a function of the Coulomb repulsion $U$ with calcium and oxygen atoms omitted for clarity. For $U>1.3 \mathrm{eV}$, ordering of dilated (large, $\mathrm{L}$ ) and contracted (small, $\mathrm{S}$ ) $\mathrm{RuO}_{6}$ octahedra occur in a rocksaltlike pattern, reducing the symmetry from $B b 2_{1} m \rightarrow P n 2_{1} a$. Change in (b) $\mathrm{RuO}_{6}$ octahedra volume $V$, (c) percent difference in volumes of two adjacent octahedra $B\left(\mathrm{RuO}_{6}\right)$, and (d) local Ru spin moment with $U+$ SOI. (e) Coulomb-dependent energy difference $\Delta E$ between the $B b 2{ }_{1} m$ and $P n 2_{1} a$ phases without SOI. 
TABLE I. Changes in local $\mathrm{RuO}_{6}$ octahedral volume $V\left(\AA^{3}\right)$, Ru$\mathrm{O}$ bond lengths $\ell(\AA)$, and $\mathrm{Ru}-\mathrm{O}-\mathrm{Ru}$ angles (average in plane, out of plane) measured in degrees with Coulomb interaction $U$. The Ru $8 b$ site in $B b 2_{1} m \mathrm{Ca}_{3} \mathrm{Ru}_{2} \mathrm{O}_{7}$ splits into two $4 a$ sites in $P n 2_{1} a$ as specified in parentheses.

\begin{tabular}{lcc}
\hline \hline & $U=0 \mathrm{eV}$ & $U=1.6 \mathrm{eV}$ \\
\hline$V\left(\mathrm{RuO}_{6}\right)$ & $10.54(8 b)$ & $10.58(4 a) ; 10.44(4 a)$ \\
$\ell(\mathrm{Ru}-\mathrm{O})$ & $1.981,1.983$ & $1.973,1.977 ; 1.971,1.979$ \\
& $1.988,1.992$ & $1.933,2.002 ; 1.986,1.990$ \\
$\angle(\mathrm{Ru}-\mathrm{O}-\mathrm{Ru})$ & $2.006,2.009$ & $2.013,2.015 ; 1.995,1.997$ \\
\hline \hline
\end{tabular}

DFT calculations have shown a strong interplay between SOI and Coulomb repulsion $U[14,15]$. For this reason, unless otherwise noted, we perform all calculations with SOI and Coulomb repulsion following the Dudarev formalism [26]. We also relax the atomic positions (forces $<0.1 \mathrm{meV}^{-1}$ ) at the DFT $+U+$ SOI level using Gaussian smearing (20-meV width), the experimental lattice parameters at $8 \mathrm{~K}$ [1], and the AFM- $b$ magnetic order.

\section{RESULTS AND DISCUSSION}

\section{A. Crystal structure}

Upon varying $U$ and performing DFT $+U+$ SOI atomic relaxations, we find that $\mathrm{Ca}_{3} \mathrm{Ru}_{2} \mathrm{O}_{7}$ undergoes a structural transition for $U>1.3 \mathrm{eV}$ characterized by a change in translational symmetry. The $B b 2{ }_{1} m$ structure with AFM- $b$ order is dynamically unstable and transforms to the primitive orthorhombic $P n 2_{1} a$ structure [Fig. 2(a)]. The polar $P n 2_{1} a$ structure is similar to the $B b 2{ }_{1} m$ phase; however, the differential Ru-O bond distortions within the octahedra (Table I), which resemble breathinglike distortions, remove the $B$ centering operation and permit a charge-density wave (CDW). The local distortions in $P n 2_{1} a$ tile in a rocksaltlike arrangement of small (S) and large (L) $\mathrm{RuO}_{6}$ octahedra [Fig. 2(a)]. For this reason, while the $B b 2_{1} m$ phase is described using two formula units, the $P n 2_{1} a$ structure is described using four formula units. Also, as indicated by the space-group symmetry, the $b$-glide and mirror planes in the $B b 2_{1} m$ structure transform to $n$ - and $a$-glide operations in the $P n 2{ }_{1} a$ phase, respectively. This subtle change in crystallography gives weak Bragg reflections of the type $(0, k, l)$, with $k, l$ being odd for $P n 2_{1} a$, that are absent in $B b 2_{1} m$. Importantly, convergentbeam electron-diffraction (CBED) experiments reported in Ref. [1] are performed in the $(h, k, 0)$ scattering plane and not $(0, k, l)$, which prohibits the reported experiment geometry from differentiating between these two space groups.

Figure 2(b) shows the octahedral volume $V$ expansion and contraction are modulated by the degree of Coulomb repulsion, which we quantify as $B\left(\mathrm{RuO}_{6}\right)$ in Fig. 2(c) using the percent difference in volumes of two adjacent $\mathrm{RuO}_{6}$. As $U$ increases in the $P n 2_{1} a$ phase, the average $\mathrm{RuO}_{6}$ octahedra volume decreases following the trend exhibited by the $B b 2_{1} m$ structure. The average in-plane and out-of-plane Ru-O-Ru angles (Table I and Ref. [27]) exhibit a small increase with $U$, which is in remarkable agreement with the measured temperature dependence below $T_{s}$ [1]. The Ca displacements that lift inversion symmetry undergo minimal changes owing to weak coupling of these displacements to the lowenergy states on the Fermi surface [28]. Importantly, we are unable to stabilize the $P n 2_{1} a$ phase with AFM- $a$ magnetic ordering, confirming the cooperation among Coulomb repulsion, magnetic ordering, and structural degrees of freedom in $\mathrm{Ca}_{3} \mathrm{Ru}_{2} \mathrm{O}_{7}$.

Coincident with the symmetry reduction, we find disproportionation of the local $\mathrm{Ru}$ spin moments, suggesting a spin-density wave (SDW) occurs along with the CDW. The small (large) $\mathrm{RuO}_{6}$ octahedra exhibits a larger (smaller) magnetic moment compared to the adjacent large (small) octahedra [Fig. 2(d)]. This difference increases with increasing $U$, reaching $\approx 0.2 \mu_{B}$ for $U=2 \mathrm{eV}$. Owing to the spin disproportionation, the magnetic space group changes from $P_{C} n a 2_{1}$ (No. 33.154, crystal class $m m 21^{\prime}$ [29]) to Pna2 ${ }_{1}$ (No. 33.144 , crystal class $m m 2$ ) [30]. Unlike the breathing distortion $\mathrm{B}\left(\mathrm{RuO}_{6}\right)$, the average magnetic moment increases with increasing Coulomb repulsion, indicating overall localization of the itinerant holes within the nominal $t_{2 g}$ manifold of $\mathrm{Ru}$. The coordinations of the small (large) $\mathrm{RuO}_{6}$ octahedra [27] suggest weak $G$-type orbital order with the $d_{x y}$ orbital lower (higher) in energy for the large (small) $\mathrm{RuO}_{6}$ octahedra. Moreover, we find a weak orbital moment of $\approx 8 \times 10^{-3} \mu_{B}$.

Spin-orbit interaction plays a key role in stabilizing the $P n 2_{1} a$ phase in the presence of the aforementioned degrees of freedom. Upon excluding SOI but allowing full atomic relaxations, we find the $P n 2_{1} a$ phase remains more stable than the $B b 2{ }_{1} m$ phase for $U>1.1 \mathrm{eV}$ [Fig. 2(e)]. For $U>1.4 \mathrm{eV}$, the total energy difference of the $P n 2_{1} a$ structures with and without SOI is $\approx 0.1 \mathrm{eV} / \mathrm{f}$.u. However, the spontaneous relaxation of $B b 2{ }_{1} m \rightarrow P n 2_{1} a$ does not occur-both phases are metastable and separated by an energy barrier, despite the onedimensional $Y_{2}$ order parameter for the symmetry-breaking displacements permitting the transition to be continuous. This can be understood as follows: The inclusion of SOI in the calculations reduces the magnetic symmetry from $C_{1 h}$ to $C_{1}$ (loss of inversion), allowing energy-lowering displacements due to the broken symmetry. To confirm this behavior, we performed a structural relaxation with $U=1.6 \mathrm{eV}$ excluding SOI starting from the $B b 2_{1} m$ phase and removing all symmetries from the calculation. We found that the $B b 2{ }_{1} m$ phase relaxes into the triclinic $P 1$ space group, which exhibits the same type of breathing distortion present in the $P n 2_{1} a$ phase but is slightly higher in energy than the $P n 2_{1} a$ ground state [27]. For these reasons, an accurate atomic ground-state description of $\mathrm{Ca}_{3} \mathrm{Ru}_{2} \mathrm{O}_{7}$ requires treating both SOI and Coulomb repulsion.

\section{B. Electronic structure}

Figure 3 shows the total density of states (DOS) of $\mathrm{Ca}_{3} \mathrm{Ru}_{2} \mathrm{O}_{7}$ as a function of the Coulomb-repulsion strength. The low-energy electronic structure consists of $\mathrm{Ru} 4 d$ states, nominally with low-spin $\mathrm{Ru}^{4+}$ in a $d^{2}(x y) d^{1}(y z) d^{1}(x z)$ configuration hybridized with $\mathrm{O} 2 p$ states [5]. For $U=0 \mathrm{eV}$, we find that the valence band is primarily $d(x y)$ with nearly full occupancy, while the conduction band consists of primarily unoccupied $d(x z)$-derived states, spanning from the Fermi level $E_{F}$ to $\approx 0.2 \mathrm{eV}$, followed by the $d(y z)$ orbitals located higher in energy $(\approx 0.2-0.4 \mathrm{eV})$. The orbital hierarchy is a 


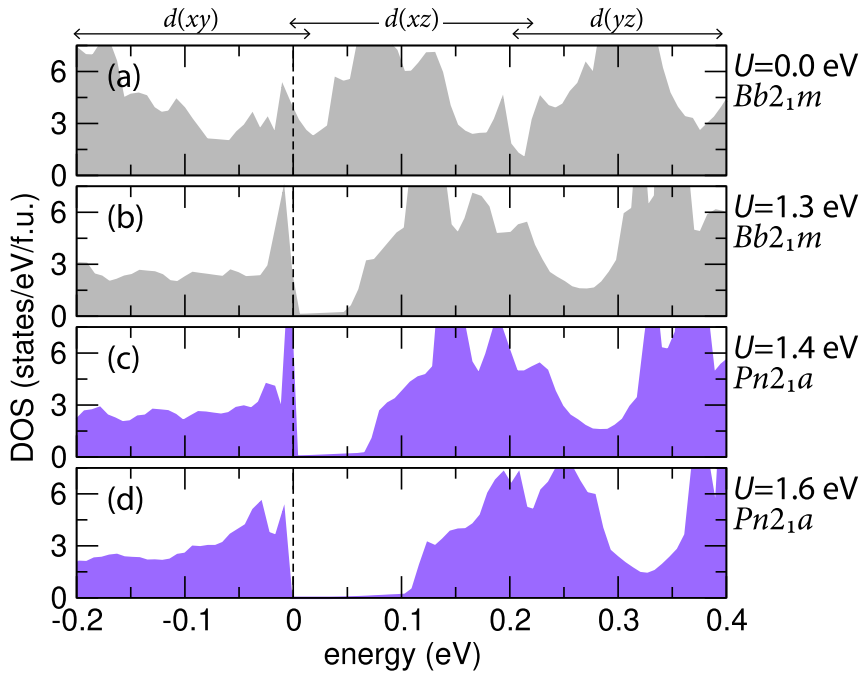

FIG. 3. DFT $+U+$ SOI calculated total density of states (DOS) for $\mathrm{Ca}_{3} \mathrm{Ru}_{2} \mathrm{O}_{7} . E_{F}=0 \mathrm{eV}$ (dashed line).

consequence of the crystal-field-split states and ratio of the lattice constants $b / a>1$; furthermore, the layered structure enhances the bandwidth of the $d(x y)$-derived bands relative to the $d(x z) / d(y z)$-derived states. As a consequence, the band structure is nearly semimetallic with multiple bands crossing $E_{F}$ and exhibits quasi-2D character (Fig. 4): The fine structure in the DOS below $E_{F}$ arises from the Ru multiplicity for the $d(x y)$-derived states, which are degenerate at $M^{\prime}(0, \pi / b)$ but split off from each other at the zone boundary $X(\pi / a, \pi / b)$, as shown in Fig. 4, and the extension of the $d(x z)$-derived bands below $E_{F}$. These features evolve with $U$, as we discuss below. SOI also lifts spin degeneracies along particular paths in the Brillouin zone due to Rashba and Dresselhaus interactions, e.g., for the aforementioned $d(x y)$-derived states spanning $E_{F}$ along the $X(\pi / a, \pi / b)-M(\pi / a, 0)$ trajectory.

Independent of the crystal structure, a metal-semimetal transition occurs as a pseudogap opens at $E_{F}$ for $U>0 \mathrm{eV}$. The width of the pseudogap depends on $U$, consistent with the temperature dependence of the pseudogap obtained from $a b$-plane optical conductivity spectra [12]. Raman scattering performed on single crystals of $\mathrm{Ca}_{3} \mathrm{Ru}_{2} \mathrm{O}_{7}$ indicate a pseudogap of $\approx 96 \mathrm{meV}$ appears at $T_{s}$ [31], suggesting that any model of the low-temperature phases of $\mathrm{Ca}_{3} \mathrm{Ru}_{2} \mathrm{O}_{7}$ should include Coulomb interactions (and SOI). The pseudogap in the $B b 2_{1} m$ phase arises from the $U$ interaction favoring preferential orbital occupancy and a redistribution of charge among the $d(x y)$ and $d(x z)$ orbitals along with small bandwidth narrowing. Indeed, while the $d(x y)$-derived bands located at $X(\pi / a, \pi / b)$ shift to lower energy with $U$, the $d(x z)$-derived bands located at $\Gamma$ [and $d(y z)$-derived states] shift to higher energy (Fig. 3 and Ref. [27]).

The SOI interaction with $U \neq 0$ increases the energy separation among fractionally occupied states relative to those that are occupied [32] because the $d(x y), d(x z)$, and $d(y z)$ states are already split by the $C_{1}$ crystal field (Table I) and the $d(x y)$ like band is close to integer filling. As a result, we observe a VHS near $E_{F}$ (Fig. 3), which is sensitive to small changes in $U$, and hence, its position and access to it should be temperature and sample purity dependent. This places $\mathrm{Ca}_{3} \mathrm{Ru}_{2} \mathrm{O}_{7}$

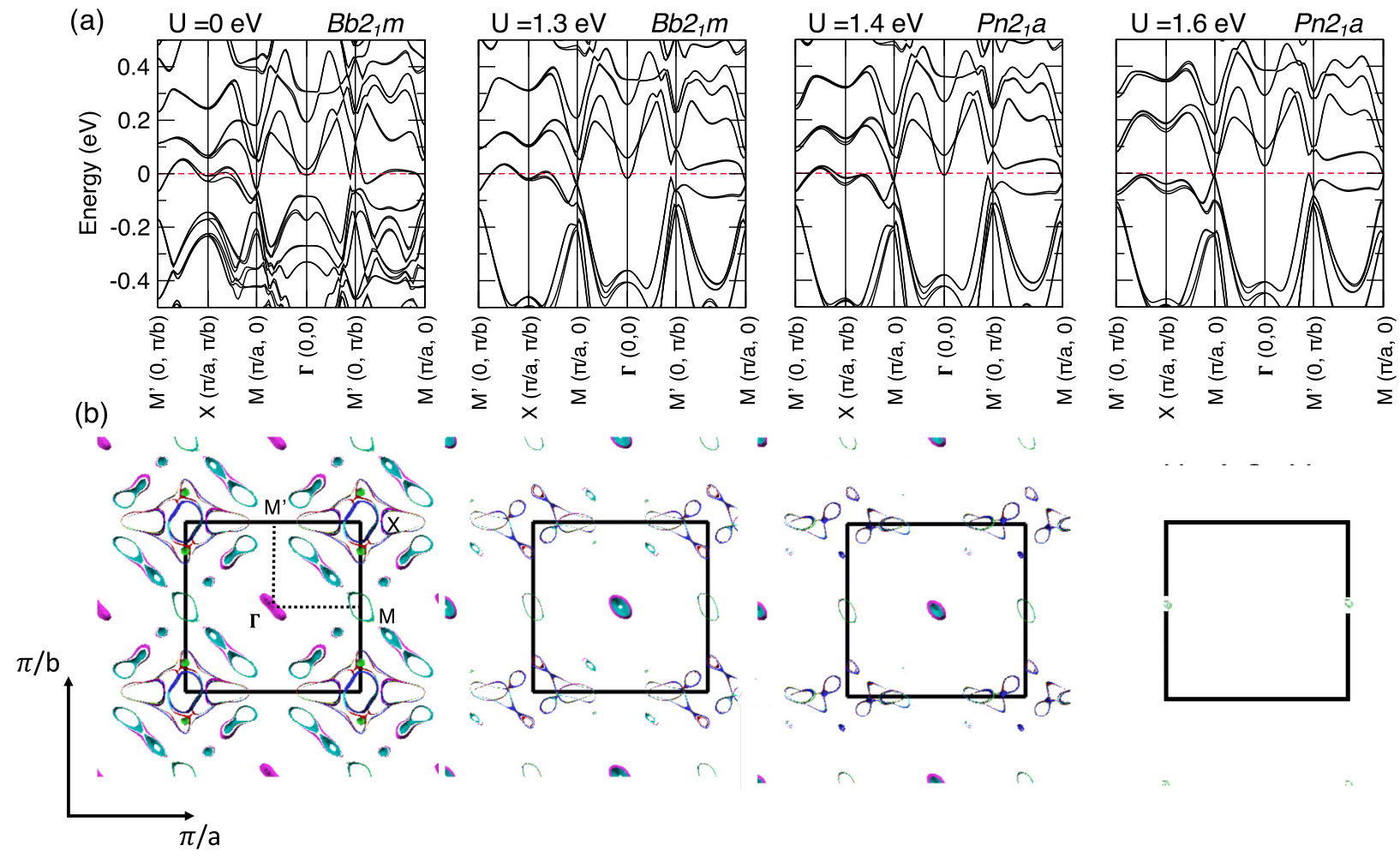

FIG. 4. DFT $+U+$ SOI calculated (a) electronic band structures and (b) Fermi surfaces in the $k_{z}=0$ plane. The Fermi level is at 0 eV (dashed line). 
proximate to an electronic instability in the $B b 2{ }_{1} m$ phase and susceptible to a Fermi surface reconstruction. For this reason, we believe the temperature-dependent thermopower data in Ref. [13] should be interpreted with caution since the sign of thermopower cannot be linked directly to the type of carrier when a VHS is within $\approx 10 \mathrm{meV}$ of $E_{F}$ [33].

To understand the origin of the VHS, we next examine in further detail the band dispersions, focusing on the $k_{z}=0$ 2D slice of the Brillouin zone (Fig. 4) because dispersions along $k_{z}$ are weak [14]. For $U=0 \mathrm{eV}$, the $\mathrm{Ru}$ bands give rise to quasi-2D hole and electron pockets along $M^{\prime}(0, \pi / b) \rightarrow X(\pi / a, \pi / b)$ with the electron pockets centered on $X(\pi / a, \pi / b)$. We find an electronlike knob at the $\Gamma$ point along with small quasi-2D electron pockets about $\Gamma$ and $M(\pi / a, 0)$. These features are clearly discernible in the FS [Fig. 4(b)], which presents small degenerate electron pockets centered at $X(\pi / a, \pi / b)$ and $M(\pi / a, 0)$, degenerate hole pockets bordering the electron pocket at $X(\pi / a, \pi / b)$, and a feature which almost connects a linear trajectory from $M(\pi / a, 0)$ to $M^{\prime}(0, \pi / b)$.

The changes in carrier type in momentum space are due to a saddle point near $E_{F}$ along the $M^{\prime}(0, \pi / b)-X(\pi / a, \pi / b)-M(\pi / a, 0)$ trajectory. In addition, nearly flat bands cross $E_{F}$ along the $M^{\prime}(0, \pi / b)-M(\pi / a, 0)$ line; beginning with $U=1.3 \mathrm{eV}$, a gap opens along this trajectory and persists (Fig. 4). The orbital-energy degeneracies within $25 \mathrm{meV}$ of $E_{F}$ at $X(\pi / a, \pi / b)$ are responsible for the identified VHS in the DOS (Fig. 3). Figure 4 also allows us to deduce that the electron pockets centered near $M(\pi / a, 0)$ originate from a Dirac-like feature, located at $\approx 70 \mathrm{meV}$ below $E_{F}$, which was recently detected by ARPES experiments [11]. These features shift away from $M(\pi / a, 0)$ owing to SOI and the broken parity and time-reversal symmetries in $\mathrm{Ca}_{3} \mathrm{Ru}_{2} \mathrm{O}_{7}$.

The change in crystal structure also affects the VHS and low-energy electronic structure for $U>1.3 \mathrm{eV}$. Figure 3(c) shows that the VHS splits just below $E_{F}$ because the change in the local $\mathrm{Ru}$ crystal field from the breathing distortions lifts the degeneracy of the single-particle $d(x y)$-derived eigenenergies from different $\mathrm{RuO}_{6}$ octahedra [see Fig. 4(a) at $X(\pi / a, \pi / b)$ orbitals]. Upon increasing $U$, these states shift to lower energy while the electron pocket derived from the $d(x z)$ orbital is fully deoccupied at $\Gamma$. The bandwidth is further reduced with increasing $U$, which also enhances the SOI spin splittings. Cooperatively, these effects shift the VHS to lower energy [Fig. 3(d)] and reduce the FS to comprise small electron pockets at $M(\pi / a, 0)$ proximate to Weyl nodes (Fig. 4).

These findings confirm the importance of SOI and Coulomb repulsion in the treatment of the low-temperature phase of $\mathrm{Ca}_{3} \mathrm{Ru}_{2} \mathrm{O}_{7}$ [14]. For $0<U<1.6 \mathrm{eV}$, the area of the Brillouin zone occupied by the FS is reduced. The volume of the electron pockets centered about $M(\pi / a, 0)$ decreases, and the features along the $M^{\prime}(0, \pi / b)-M(\pi / a, 0)$ line and at the $\Gamma$ point vanish. Due to the shift of the VHS, the electron pockets centered on $X(\pi / a, \pi / b)$ also disappear, while the nearby hole pockets survive. For $U=1.6 \mathrm{eV}$, only the electron pockets about $M(\pi / a, 0)$ survive-all bands are gapped with the exception of the Dirac-like structure that shifts to $\sim 15 \mathrm{meV}$ below $E_{F}$. In addition, we find that a full charge gap $(\approx 30 \mathrm{meV})$ appears for $U=1.8 \mathrm{eV}$ with SOI. This critical value of $U$ for the metal-insulator transition in $\mathrm{Ca}_{3} \mathrm{Ru}_{2} \mathrm{O}_{7}$ is well below $U=3 \mathrm{eV}$ reported in Ref. [14], which did not allow for local changes in the atomic structure at the DFT $+U+$ SOI level. Importantly, no insulating state is reported for $\mathrm{Ca}_{3} \mathrm{Ru}_{2} \mathrm{O}_{7}$; hence, $U=1.8 \mathrm{eV}$ is the upper bound on the interaction strength within our model.

\section{Phase transition assignments}

The addition of Coulomb repulsion within our DFT calculations has three major effects: it (i) shifts the position of the VHS to lower energy, (ii) reduces the overlap between the top of the valence band and the bottom of the conduction band, and (iii) shifts the Dirac-like feature at $M(\pi / a, 0)$ to higher energy. Therefore, it is natural to ask what is the correct value for $U$ and how it can be used to model the temperaturedependent fermiology. To this purpose, we now compare our calculated electronic band structures as a function of $U+$ SOI with ARPES-measured dispersions at $16 \mathrm{~K}$ [11]. We find best agreement for $U=1.6 \mathrm{eV}$ (Fig. 5). In particular, the Dirac-like feature at $(\pi / a, 0)$ and the electronic band structure along $\Gamma-M^{\prime}(0, \pi / b)$ are well captured. Note that for $U>$ $1.3 \mathrm{eV}$, we predict a change in symmetry $\left(B b 2{ }_{1} m \rightarrow P n 2_{1} a\right)$ for $\mathrm{Ca}_{3} \mathrm{Ru}_{2} \mathrm{O}_{7}$. Using this as an approximate bound on the ground-state $U$ value for $\mathrm{Ca}_{3} \mathrm{Ru}_{2} \mathrm{O}_{7}$, we assign $T=30 \mathrm{~K}$ to be a CDW-SDW transition.

Furthermore, reasonable values of $U$ to model intermediate temperatures $T_{\mathrm{CDW}}=30<T<T_{S}=48 \mathrm{~K}$ then should span values of $0.5<U<1.6 \mathrm{eV}$. This is justified by the increase of the pseudogap with temperature revealed by optical spectroscopy [12]. For this range of Coulomb interactions, we find

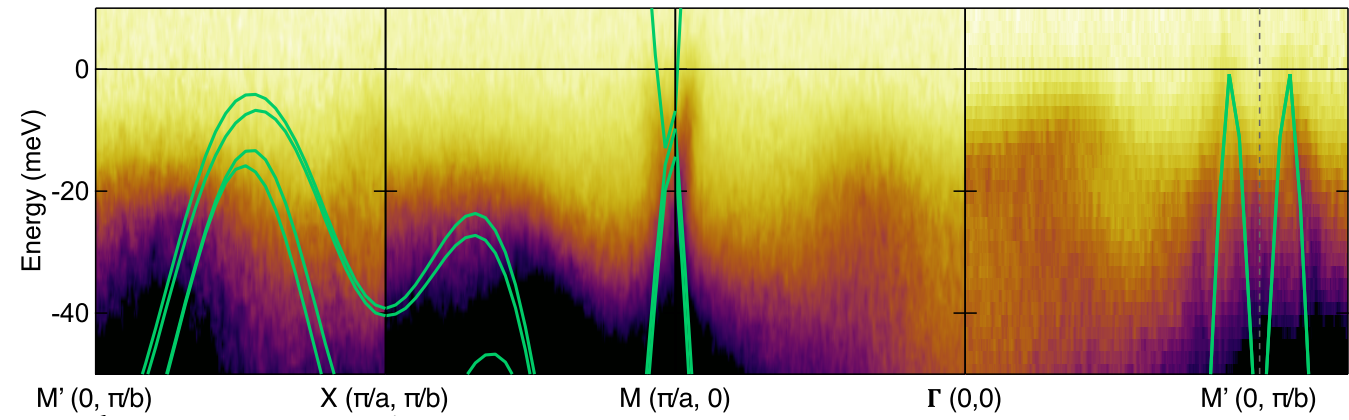

FIG. 5. Comparison of the DFT $+U+\mathrm{SOI}(U=1.6 \mathrm{eV})$ band dispersions with those from Ref. [11] acquired at $16 \mathrm{~K}$. 
that weak static correlations with $\mathrm{SOI}$ and $\mathrm{RuO}_{6}$ distortions drive a Lifshitz-like transition that reconstructs the Fermi surface and opens a pseudogap at $T_{s}$ due to filling of the $d(x y)$ bands preferentially to the $d(x z)$ band that is emptied, leaving behind a VHS and Weyl nodes just below $E_{F}$. In this temperature range, it is plausible that the $P n 2_{1} a$ phase persists; further experiments are required to conclusively resolve this conjecture.

\section{CONCLUSIONS}

Using DFT calculations combined with ARPES measurements, we reconciled existing experiments on the lowtemperature fermiology of $\mathrm{Ca}_{3} \mathrm{Ru}_{2} \mathrm{O}_{7}$. The cooperative interplay among Coulomb repulsion $U$, magnetic ordering, spin-orbit interactions, and structural degrees of freedom governs the temperature-dependent phase transitions. We assigned a Lifshitz-like transition to $T_{s}=48 \mathrm{~K}$ and a charge(spin-) density wave transition to $T_{\mathrm{CDW}}=30 \mathrm{~K}$, which were hypothesized from optical spectroscopy [12], along with broken translational symmetry $\left(B b 2_{1} m \rightarrow P n 2_{1} a\right)$ at $T_{\mathrm{CDW}}$. Last, we validated our temperature-dependent Coulomb interaction model with ARPES experiments at $16 \mathrm{~K}$ [11], which indicated that the low-energy physics requires moderate Coulomb repulsion $(U=1.6 \mathrm{eV})$ and the unique $P n 2{ }_{1} a$ symmetry. We hope new structural assessments are performed at low temperature with advanced CBED or synchrotron scattering techniques to assess the predicted revised phase diagram. Our work points to the importance of treating multiple interactions on equal footing in correlated metals, which exhibit nontrivial fermions near the Fermi level and fractional orbital filling, as SOI and Coulombic interactions may conspire with lattice and spin degrees of freedom to produce unexpected electronic and structural transitions.

\section{ACKNOWLEDGMENTS}

M.H. and J.C. thank M. H. Fischer for insightful discussions. D.P. and J.M.R. acknowledge the Army Research Office under Grant No. W911NF-15-1-0017 for financial support and the DOD-HPCMP for computational resources. M.H. and J.C. acknowledge support from the Swiss National Science Foundation.
[1] Y. Yoshida, S.-I. Ikeda, H. Matsuhata, N. Shirakawa, C. H. Lee, and S. Katano, Phys. Rev. B 72, 054412 (2005).

[2] G. Cao, L. Balicas, Y. Xin, E. Dagotto, J. E. Crow, C. S. Nelson, and D. F. Agterberg, Phys. Rev. B 67, 060406(R) (2003).

[3] X. N. Lin, Z. X. Zhou, V. Durairaj, P. Schlottmann, and G. Cao, Phys. Rev. Lett. 95, 017203 (2005).

[4] G. Cao, X. N. Lin, L. Balicas, S. Chikara, J. E. Crow, and P. Schlottmann, New J. Phys. 6, 159 (2004).

[5] S. Lei, M. Gu, D. Puggioni, G. Stone, J. Peng, J. Ge, Y. Wang, B. Wang, Y. Yuan, K. Wang, Z. Mao, J. M. Rondinelli, and V. Gopalan, Nano Lett. 18, 3088 (2018); G. Stone, D. Puggioni, S. Lei, M. Gu, K. Wang, Y. Wang, J. Ge, X.-Z. Lu, Z. Mao, J. M. Rondinelli, and V. Gopalan, Phys. Rev. B 99, 014105 (2019).

[6] A. G. Christy, Acta Crystallogr., Sect. B 51, 753 (1995).

[7] D. N. Basov, R. D. Averitt, and D. Hsieh, Nat. Mater. 16, 1077 (2017).

[8] F. Baumberger, N. J. C. Ingle, N. Kikugawa, M. A. Hossain, W. Meevasana, R. S. Perry, K. M. Shen, D. H. Lu, A. Damascelli, A. Rost, A. P. Mackenzie, Z. Hussain, and Z.-X. Shen, Phys. Rev. Lett. 96, 107601 (2006).

[9] I. Marković, M. D. Watson, O. J. Clark, F. Mazzola, E. A. Morales, C. A. Hooley, H. Rosner, C. M. Polley, T. Balasubramanian, S. Mukherjee, N. Kikugawa, D. A. Sokolov, A. P. Mackenzie, and P. D. C. King, arXiv:2001.09499.

[10] Y. Yuan, P. Kissin, D. Puggioni, K. Cremin, S. Lei, Y. Wang, Z. Mao, J. M. Rondinelli, R. D. Averitt, and V. Gopalan, Phys. Rev. B 99, 155111 (2019).

[11] M. Horio, Q. Wang, V. Granata, K. P. Kramer, Y. Sassa, S. Jöhr, D. Sutter, A. Bold, L. Das, Y. Xu, R. Frison, R. Fittipaldi, T. K. Kim, C. Cacho, J. E. Rault, P. L. Févre, F. Bertran, N. C. Plumb, M. Shi, A. Vecchione, M. H. Fischer, and J. Chang, arXiv:1911.12163.
[12] J. S. Lee, S. J. Moon, B. J. Yang, J. Yu, U. Schade, Y. Yoshida, S.-I. Ikeda, and T. W. Noh, Phys. Rev. Lett. 98, 097403 (2007).

[13] H. Xing, L. Wen, C. Shen, J. He, X. Cai, J. Peng, S. Wang, M. Tian, Z.-A. Xu, W. Ku, Z. Mao, and Y. Liu, Phys. Rev. B 97, 041113(R) (2018).

[14] G.-Q. Liu, Phys. Rev. B 84, 235137 (2011).

[15] Z. Jin and W. Ku, arXiv:1809.04589.

[16] G. Gou, I. Grinberg, A. M. Rappe, and J. M. Rondinelli, Phys. Rev. B 84, 144101 (2011).

[17] S. Grebinskij, Š. Masys, S. Mickevičius, V. Lisauskas, and V. Jonauskas, Phys. Rev. B 87, 035106 (2013).

[18] C. Etz, I. V. Maznichenko, D. Böttcher, J. Henk, A. N. Yaresko, W. Hergert, I. I. Mazin, I. Mertig, and A. Ernst, Phys. Rev. B 86, 064441 (2012).

[19] H. Akamatsu, K. Fujita, T. Kuge, A. Sen Gupta, A. Togo, S. Lei, F. Xue, G. Stone, J. M. Rondinelli, L.-Q. Chen, I. Tanaka, V. Gopalan, and K. Tanaka, Phys. Rev. Lett. 112, 187602 (2014).

[20] T. Zhu, T. Cohen, A. S. Gibbs, W. Zhang, P. S. Halasyamani, M. A. Hayward, and N. A. Benedek, Chem. Mater. 29, 9489 (2017).

[21] L. Fratino, P. Sémon, M. Charlebois, G. Sordi, and A.-M. S. Tremblay, Phys. Rev. B 95, 235109 (2017); K. Seki, T. Shirakawa, and S. Yunoki, ibid. 98, 205114 (2018).

[22] We use the Perdew-Burke-Ernzerhof exchange-correlation functional revised for solids (PBEsol) [34] as implemented in the Vienna Ab initio Simulation Package (VASP) [35] with the projector augmented-wave approach [36] to treat the core and valence electrons using the following electronic configurations: $3 s^{2} 3 p^{6} 4 s^{2}(\mathrm{Ca}), 4 d^{7} 5 s^{1}(\mathrm{Ru})$, and $2 s^{2} 2 p^{4}(\mathrm{O})$, with a $5 \times 5 \times 3$ Monkhorst-Pack $k$-point mesh [37] and a $650-\mathrm{eV}$ plane-wave cutoff.

[23] G. Zhang and E. Pavarini, Phys. Rev. B 95, 075145 (2017). 
[24] D. Sutter, C. G. Fatuzzo, S. Moser, M. Kim, R. Fittipaldi, A. Vecchione, V. Granata, Y. Sassa, F. Cossalter, G. Gatti, M. Grioni, H. M. Rønnow, N. C. Plumb, C. E. Matt, M. Shi, M. Hoesch, T. K. Kim, T.-R. Chang, H.-T. Jeng, C. Jozwiak, A. Bostwick, E. Rotenberg, A. Georges, T. Neupert, and J. Chang, Nat. Commun. 8, 15176 (2017).

[25] L. Das, F. Forte, R. Fittipaldi, C. G. Fatuzzo, V. Granata, O. Ivashko, M. Horio, F. Schindler, M. Dantz, Y. Tseng, D. E. McNally, H. M. Rønnow, W. Wan, N. B. Christensen, J. Pelliciari, P. Olalde-Velasco, N. Kikugawa, T. Neupert, A. Vecchione, T. Schmitt, M. Cuoco, and J. Chang, Phys. Rev. X 8, 011048 (2018).

[26] S. L. Dudarev, G. A. Botton, S. Y. Savrasov, C. J. Humphreys, and A. P. Sutton, Phys. Rev. B 57, 1505 (1998).

[27] See Supplemental Material at http://link.aps.org/supplemental/ 10.1103/PhysRevResearch.2.023141 for additional structural data and electronic structures.

[28] D. Puggioni and J. M. Rondinelli, Nat. Commun. 5, 3432 (2014).
[29] S. W. Lovesey, D. D. Khalyavin, and G. van der Laan, Phys. Rev. B 99, 134444 (2019).

[30] We use the Belov-Neronova-Smirnova setting of magnetic space groups; see the Bilbao Crystallographic server, http:// www.cryst.ehu.es.

[31] H. L. Liu, S. Yoon, S. L. Cooper, G. Cao, and J. E. Crow, Phys. Rev. B 60, R6980 (1999).

[32] G. Zhang and E. Pavarini, Phys. Status Solidi RRL 12, 1800211 (2018).

[33] G. C. McIntosh and A. B. Kaiser, Phys. Rev. B 54, 12569 (1996).

[34] J. P. Perdew, A. Ruzsinszky, G. I. Csonka, O. A. Vydrov, G. E. Scuseria, L. A. Constantin, X. Zhou, and K. Burke, Phys. Rev. Lett. 100, 136406 (2008).

[35] G. Kresse and J. Furthmüller, Comput. Mater. Sci. 6, 15 (1996).

[36] P. E. Blöchl, O. Jepsen, and O. K. Andersen, Phys. Rev. B 49, 16223 (1994).

[37] H. J. Monkhorst and J. D. Pack, Phys. Rev. B 13, 5188 (1976). 